\title{
Performances de croissance, de ponte et qualité de l'œuf chez la caille Japonaise (Coturnix coturnix japonica)
}

\author{
Ouaffai, A.; Dahloum, L. @ ; Fassih, A.; Milagh, M. et Halbouche, M.
}

Laboratoire de Physiologie Animale Appliquée (LPAA). Université Abdelhamid Ibn Badis de Mostaganem UMAB. Algérie.

\section{MOTS CLÉS SUPPLÉMENTAIRES}

Coturnix coturnix japonica.

Croissance.

Performanace de ponte.

Qualité des œufs.

Corrélations phénotypiques.

\section{RÉSUMÉ}

Le présent travail a consisté à étudier les performances de croissance (durant 6 semaines) et de ponte (9ème-12ème semaine) de Coturnix coturnix japonica ainsi que la qualité interne et externe des œufs. Au total, 300 cailleteaux de 1 jour d'âge ont été menés au sol. Á 6 semaines d'âge, le poids vif (PV) moyen a été de 162g avec un indice de consommation de 3,59. Les mensurations effectuées à l'âge de 8 semaines ont montré un dimorphisme sexuel significatif $(P<0,05)$ en faveur des femelles pour le poids vif, la longueur du corps, l'envergure et la largeur du thorax. L'âge moyen au premier œuf a été de 49 jours. Le taux de ponte enregistré a été de $52,1 \%$, soit une production moyenne de 14,6 œuf/femelle. Le taux d'éclosion et la viabilité ont été respectivement de $84,5 \%$ et $76,5 \%$. Le poids moyen de l'œuf entier a été de $11,5 \mathrm{~g}$ avec un indice de forme moyen de 78,2 . Le poids de la coquille, celui du blanc et celui du vitellus ont été respectivement de 1,$47 ; 5,81$ et $3,5 \mathrm{~g}$; ceci correspond à des proportions (par rapport au poids de l'œuf entier) respectivement de 12,$8 ; 50,3$ et $30,4 \%$. Les unités Haugh (UH) ont été de 57,4 , en moyenne. Les femelles maintenues à faible densité (20 oiseaux/cage) ont produit des œufs plus lourds $(P<0,001)$ et plus larges $(\mathrm{P}<0,01)$ que ceux pondus dans les cages à plus forte densité (25 oiseaux/cage). Par contre, ce dernier groupe a manifesté sa supériorité en termes des UH et des proportions des trois constituants (coquille, blanc et jaune). L'analyse en composantes principales (ACP) appliquée pour les neuf caractéristiques de l'œuf de caille a permis d'obtenir 3 composantes (CP1, CP2 et CP3) expliquant respectivement $44,3 \%, 14,2 \%$ et $11,9 \%$, soit un pourcentage cumulé de $70,5 \%$ de la variance totale. Le dernier axe (CP3) est considéré comme facteur spécifique pour le poids de la coquille.

\section{Growth, Laying Performance and Egg Quality Traits in Japanese Quail (Coturnix coturnix japonica)}

\section{SUMMARY}

\section{ADDITIONAL KEYWORDS}

Coturnix coturnix japonica.

Growth.

Laying performance.

Egg quality.

Phenotypic correlations.

\section{INFORMATION}

Cronología del artículo.

Recibido/Received: 30.10 .2016

Aceptado/Accepted: 22.02.2018

On-line: 15.04 .2018

Correspondencia a los autores/Contact e-mail:

lahouari.dahloum@univ-mosta.dz

\section{INTRODUCTION}

Les volailles présentent beaucoup de caractéristiques intéressantes pour le sélectionneur. L'intensification de la production a permis le développement d'une aviculture industrielle à vocation internationale (Tixier-Boichard, 1992). La filière avicole a fourni en 2012, dans le monde, 103 millions de
The present study was conducted to evaluate the growth performance (from 1day to 6 week old), egg production (from 9 to 12 week of age) and egg quality in the Japanese quail (Coturnix coturnix japonica). A total of 300, 1-day-old Japanese quail chicks were used in this trial. Average body weight and feed intake at day 42 were $162 \mathrm{~g}$ and $553 \mathrm{~g} /$ bird, respectively, which corresponds to a feed conversion ratio of 3,59 . At 8 weeks old, live body weight, body lenght, wingspan and chest width showed a significant sexual dimorphism in favour of females. The age of the quail at first egg was 49 days. Egg production was $52,1 \%$ corresponding to 14,6 eggs/quail. Average egg weight was $11,5 \mathrm{~g}$, while hatchability and viability were $84,5 \%$ and $76,5 \%$, respectively. In terms of external and internal egg quality, the average egg weight was $11,5 \mathrm{~g}$ while the shape index was 78,2 . Eggshell weight, albumen weight and yolk weight were 1,$47 ; 5,81$ et $3,5 \mathrm{~g}$, respectively, correponding to proportions of 12,$8 ; 50,3$ and $30,4 \%$, respectively. The mean value of Haugh units (UH), indicator of egg freshness was 57,4 . Quails maintained at low density (20 birds per cage) had significantly highest egg weight and egg width than those kept at density of 25 birds per cage. The opposite has been observed in terms of $\mathrm{UH}$ and proportions of eggshell, albumen and yolk. Principal Components Analysis (PCA) with 9 egg quality traits revealed three principal components with eigen value higher than 1 . $P C 1$, PC2 and PC3 accounted for 44,3\%, 14,2\% and $11,9 \%$ of total variance, respectively, which correspond to a cumulative percentage of $70,5 \%$. The last axis (CP3) is considered as a specific factor for the eggshell.

tonnes de viande et 66,4 millions de tonnes d'œufs de consommation (Dahloum, 2017). Selon les estimations de la FAO, d'ici 2050, les besoins alimentaires de la population mondiale augmentera de $70 \%$ (FAO, 2009). Pour répondre à cette importante demande, l'élevage d'insectes et d'animaux à cycle court, la caille par exemple, peuvent présenter un atout important pour subvenir aux besoins en protéines animales et 
réduire, par conséquent, la pression sur la production du poulet.

Il est actuellement couramment admis que la domestication de la caille a commencé au Japon, les premières traces écrites à ce sujet remontant au XIIème siècle, principalement dans un but ornemental, les cailles étant alors sélectionnées pour leur chant (Recoquillay, 2014). Ce n'est qu'à la fin de la seconde guerre mondiale qu'une sélection de cailles japonaises basée sur la production de viande et d'œufs a été lancée dans ce pays. Par ailleurs, la caille japonaise a été introduite aux USA, pour la première fois, vers 1950 (Berto et al., 2013), et vers 1980 en Afrique subsaharienne (Hantanirina et al., 2013) ; pendant cette période, l'élevage de la caille fut très répandu en Europe (Berto et al., 2013). La maîtrise de son exploitation a dès lors connu des avancées au fil du temps. L'amélioration de l'élevage des cailles a été entrepris en vue de la mise à profit de leurs caractéristiques notamment la précocité de l'oiseau en production de viande (5 semaines d'âge), sa performance de ponte (200 à 300 œufs par an) et son intervalle de génération assez court, par rapport à d'autres espèces aviaires. Outre son intérêt économique, la caille a été rapidement adoptée comme modèle animal dans la recherche scientifique, physiologie animale, génétique et neurosciences comportementales, en particulier (Ferrouk et al., 2015 ; Le Bot, 2014). La caille japonaise présente des caractéristiques comportementales et physiologiques proches de celles de la poule domestique (Gallus gallus) ainsi que des conditions d'élevage similaires (Schweitzer, 2009). A la différence du poulet, néanmoins, où les schémas de sélection sont très ciblés, la sélection de la caille est très souvent effectuée d'une façon massale. Ainsi, selon les objectifs, chaque élevage a développé une souche dont les caractéristiques lui sont propres, ce qui explique, la grande hétérogénéité des populations et des performances zootechniques (Berrama et al., 2011). En Algérie, à notre connaissance, très peu d'études (Berrama et al., 2011 ; Moula et al., 2014 ; Ferrouk et al., 2015) ont été menées sur la caille Japonaise (Coturnix coturnix japonica). De ce fait, l'objectif de cette étude a été de contribuer à la diffusion d'informations complémentaires sur cette espèce en termes de performances de croissance, de ponte, et de la qualité des œufs.

\section{MATÉRIEL ET MÉTHODES}

\section{Croissance pondérale et paramètres mORPhologiques}

\section{ANIMAUXX, ALIMENTS ET CONDITIONS EXPÉRIMENTALES}

Cette étude a été pour l'essentiel menée au Centre Cynégétique de Zéralda (CCZ), et le Laboratoire de Physiologie Animale Appliquée (LPAA), de 1’Université de Mostaganem (Algérie). Au total, 300 cailleteaux de type Coturnix coturnix japonica âgés de 1 jour provenant du CCZ ont été pesés et menés au sol. Les températures moyennes ont été de $39^{\circ} \mathrm{C}$ durant la première semaine; $36^{\circ} \mathrm{C}$ durant la phase de croissance et $24^{\circ} \mathrm{C}$ durant la phase de finition. Un éclairage continu a été appliqué durant les deux premières semaines. L'aliment distribué (tableau I) était à 20\% de protéines brutes (PB) et $2900 \mathrm{Kcal} / \mathrm{Kg}$ d'énergie métabolisable (EM) en phase de démarrage-croissance, et 18\% de
PB et $2850 \mathrm{Kcal} / \mathrm{Kg}$ d'EM durant la phase de finition. Les animaux ont été nourris à volonté. Un programme prophylactique anti-stress, anti-coccidiose (Amprol) et hepatoprotecteur a été appliqué. Le cycle d'élevage a duré 6 semaines.

\section{PARAMÈTRES MESURÉS}

Quarante cinq (45) cailleteaux ont été pesés à la fin de chaque semaine d'élevage. Le taux de mortalité, la consommation alimentaire, le gain de poids quotidien (GMQ) et l'indice de consommation (IC) ont été déterminés. A l'âge de 8 semaines, le poids vif et certaines mensurations corporelles, longueur du corps $(\mathrm{LC}, \mathrm{cm})$, envergure (ENV, cm), largeur thoracique (LT, $\mathrm{cm})$, longueur des pattes (LP, cm) et longueur du bec (LB, cm) ont été effectuées sur 100 oiseaux (42 mâles et 58 femelles).

\section{PONTE, QUALITÉ DES OEUFS ET PARAMÈTRE D'INCUBATION}

\section{ANIMAUX, ALIMENT ET DISPOSITIF}

A l'entrée en ponte, à l'âge de 49 jours, 65 cailles ont été réparties dans 3 cages ( 2 cages comportant chacune 15 femelles et 5 mâles; 1 cage avec 20 femelles et 5 mâles, soit un sexe ratio $=5$ femelles $/$ mâle). La température moyenne dans $1^{\prime}$ enceinte a été de $27^{\circ} \mathrm{C}$ et $70 \% \mathrm{HR}$. Les animaux ont reçu un aliment commercial de type ponte jusqu'à la fin du cycle de production (vers 41 semaines d'âge). Le régime dosant 20,6\% de PB est composé principalement de maïs, soja, calcaire, phosphate et CMV et apporte prés de $3000 \mathrm{Kcal}$ /Kg d'EM. Le rationnement a été appliqué selon le guide d'élevage de la caille établi par 1'ITELV (Institut Techniques des Elevages de Baba Ali, Algerie). Nous ne présenterons ici que les performances de ponte enregistrées sur une période de 4 semaines (9 -12 semaines). Des résultats plus détaillés seront publiés ultérieurement.

\section{Paramettres mesurés}

Les œufs (3œufs/cage/jour) ont été ramassés quotidiennement, nettoyés et conservés pour être analysés en laboratoire dans un délai de trois jours maximum. Au total, 150 œufs ont été utilisés pour déterminer leur qualité interne et externe. Après numérotation, les œufs ont été pesés individuellement (Pércision: 0,1g). La longueur et la largeur des œufs ont été mesurées à l'aide d'un pied à coulisse (Pércision: 0,01mm). Après cassage, les constituants de l'œuf ont été déposés sur une surface plane. La hauteur du blanc (le plus épais) a été déterminée à l'aide d'une règle graduée placée à mi-distance du contour du vitellus. La hauteur du jaune a été déterminée en plaçant la règle verticalement derrière celui-ci (Angrand, 1986). Après séparation, le poids de l'albumen et celui du jaune ont été mesurés avec une balance électronique (Précision: 0,01g). $\mathrm{D}$ 'autres variables ont également été calculées:

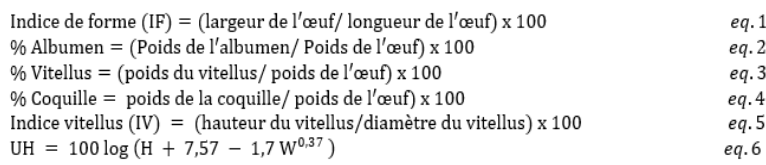


Tableau I. Composition des rations distribuées aux cailleteaux (Composition of rations distributed to quail chicks).

\begin{tabular}{lcc}
\hline Structure (\%) & $\begin{array}{c}\text { Démarrage- } \\
\text { Croissance }\end{array}$ & Finition \\
\hline Maïs & 48,50 & 59,20 \\
Tourteaux de Soja & 45,50 & 33,40 \\
Lysine & 0.05 & 0,12 \\
Calcaire & 0,75 & 0,88 \\
Phosphate bi-calcique & 1,20 & 1,10 \\
Enzyme & 0,10 & 0,10 \\
Phytase & 0,08 & 0,01 \\
Complément minéral vitaminé, CMV & 1,26 & 1,24 \\
Bentonite & 2,60 & 3,94 \\
Chlorure de Sodium & 0,01 & 0,01 \\
\hline
\end{tabular}

La durée moyenne de stockage des œufs a été de 10 jours à température ambiante $\left(24^{\circ} \mathrm{C}\right.$, en moyenne). Les œufs ont été mis en incubation pendant 17 jours $\left(37,5^{\circ} \mathrm{C}\right.$ et $\left.60 \% \mathrm{HR}\right)$ avec un retournement chaque 8 heures avant d'être transférés dans les éclosoirs $\left(38^{\circ} \mathrm{C}\right.$, $70 \% \mathrm{HR}$ ). A la naissance, 30 cailleteaux ont été pesés pour déterminer leur poids. La fertilité et la mortalité embryonnaire n'ont pas été évaluées dans cette étude.

\section{CALCULS ET TRAITEMENTS STATISTIQUES DES DONNÉES}

Les statistiques descriptives (moyenne, écart-type et coefficient de variation) ont été calculées. Le test de Student a été appliqué pour comparer les moyennes. Les coefficients de corrélation de Pearson ont été calculés pour mesurer les relations entre paramètres. Une analyse en composante principale (ACP) a été réalisée sur les paramètres de l'œuf uniquement. Dans le cas des animaux, l'indice KMO (Kaiser-Mayer-Olkin) était au-dessous de la valeur de 0,5 recommandée pour la fiabilité de l'analyse. Les données ont été traitées à l'aide du logiciel Software SPSS, version 20.

\section{RÉSULTATS ET DISCUSSION}

\section{Croissance de Coturnix coturnix japonica et MENSURATION CORPORELLES}

Les performances de croissance enregistrées chez le cailleteau durant 6 semaines d'élevage sont présentées dans le tableau II et les figures 1, 2 et 3 . Indépendamment du sexe, le cailleteaux a consommé durant cette période prés de $553 \mathrm{~g}$ d'aliments pour produire $162,4 \mathrm{~g}$ de poids vif, soit un indice de consommation de 3,59. Nos animaux ont montré, en effet, des performances bien meilleures que celles rapportées précédemment par Berrama et al. (2011); ces auteurs ont observé chez le cailleteau, une efficacité alimentaire excessivement faible, en particulier à la 6ème semaine d'âge $(\mathrm{IC}=9,6)$. Djouvinov et Mihailov (2005) situent les performances de poids vif du cailleteaux entre 146 et $175 \mathrm{~g}$ avec des indices de consommation allant de 3,49 à 4,17. Bagh et al. (2016) ont obtenu des poids vifs allant de 168,2 et $173,8 \mathrm{~g}$ chez trois variétés différentes de Cotrurnix japonica. Au même âge, Nasrollah, (2009) rapporte un poids vif de $177,5 \mathrm{~g}$ et un IC de 3,38. Des poids vifs plus élevés respectivement de 242,1 et $284,9 \mathrm{~g}$ ont été

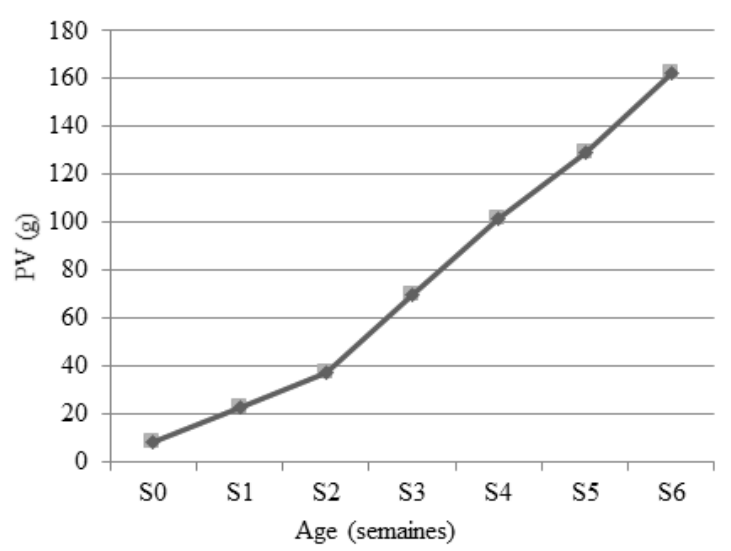

Figure 1. Evolution du poids vif de Coturnix coturnix japonica en croissance (Evolution of the live weight of Coturnix coturnix japonica in growth).

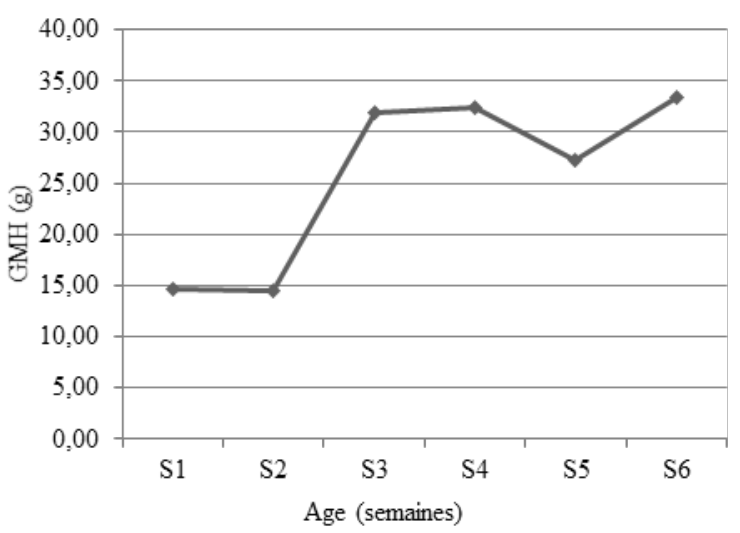

Figure 2. Evolution du gain de poids hebdomadaire de Coturnix coturnix japonica en croissance (Evolution of weekly weight gain of Coturnix Coturnix japonica in growth)

Tableau II. Performances d'élevage des cailleteaux Coturnix coturnix japonica par phase d'élevage) (Moyenne \pm écart-type) (Growth performances of Coturnix coturnix japonica chick per growth stage) (Mean \pm SD).

\begin{tabular}{|c|c|c|c|}
\hline & $\begin{array}{c}\text { Démarrage, } \\
\text { Croissance } \\
(1-21 \mathrm{j})\end{array}$ & $\begin{array}{l}\text { Finition } \\
(22-42 \mathrm{j})\end{array}$ & $\begin{array}{l}\text { Total cycle } \\
\text { (1-42 j) }\end{array}$ \\
\hline Mortalité \% & 4,02 & 0,34 & 4,36 \\
\hline $\begin{array}{l}\text { Consommation } \\
\text { alimentaire } \mathrm{g} / \mathrm{sujet}\end{array}$ & $219 \pm 7,51$ & $334,0 \pm 15,50$ & $553 \pm 20,50$ \\
\hline $\begin{array}{l}\text { Consommation } \\
\text { alimentaire } g / j\end{array}$ & $10,45 \pm 1,55$ & $15,92 \pm 1,90$ & $13,25 \pm 1,40$ \\
\hline $\begin{array}{l}\text { Poids vif de fin de } \\
\text { phase } \\
\text { g/sujet }\end{array}$ & $69,40 \pm 3,05$ & $162,4 \pm 7,30$ & $162,4 \pm 12,70$ \\
\hline $\begin{array}{l}\text { Gain de poids g/ } \\
\text { phase }\end{array}$ & $61,12 \pm 3,04$ & $93,03 \pm 5,40$ & $154,13 \pm 7,15$ \\
\hline GMQ g/j & $2,91 \pm 0,20$ & $4,43 \pm 0,80$ & $3,69 \pm 0,70$ \\
\hline $\begin{array}{l}\text { Indice de } \\
\text { consommation } \mathrm{g} / \mathrm{g}\end{array}$ & $3,58 \pm 0,15$ & $3,59 \pm 0,20$ & $3,59 \pm 0,30$ \\
\hline
\end{tabular}




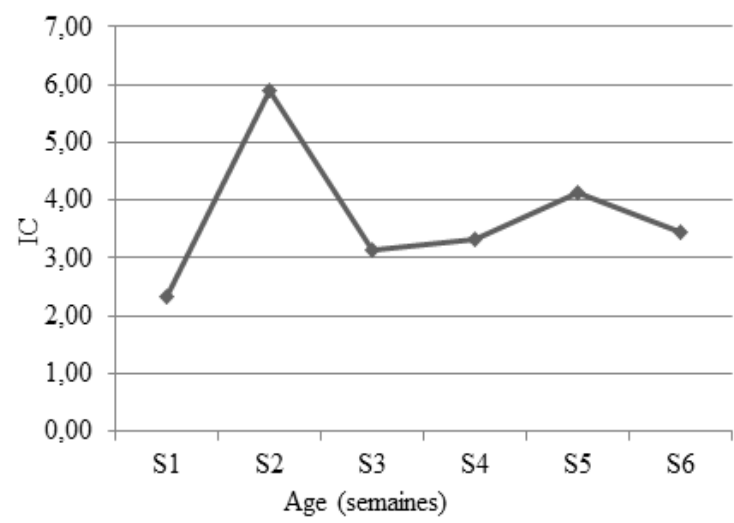

Figure 3. Indices de consommation de Coturnix coturnix japonica en croissance (Consumption indices of Coturnix Coturnix japonica in growth).

enregistrés à la 4ème et la 5ème semaine d'âge (Alasahan et Copur, 2016). Des valeurs encore bien meilleures allant de 272,2 à 302,8 g ont également été enregistrées chez 4 souches différentes de Coturnix japonica (Jatoi et al., 2017). Dans une étude menée à Madagascar, les femelles de deux souches différentes de Coturnix japonica (Grise et Jaune) ont atteint un poids vif moyen de 331g à l'âge de 6 semaines (Hantanirina et al., 2013). En revanche, chez la caille menée en cage, Narinc et al. (2010) ont rapporté un poids vif moyen de 200,5 g et un GMQ de 3,91 g/j.

Il est difficile, bien entendu, de comparer entre les résultats de ces études du fait de l'influence plus ou moins forte des facteurs génétiques, d'élevage (rationnement alimentaire notamment) et éventuellement de leurs interactions pouvant conduire à des observations opposées. Dans l'étude de Sharifi et al. (2011), ces auteurs ont observé des performances (PV, GMQ et IC)

\begin{tabular}{|c|c|c|c|c|}
\hline $\begin{array}{l}\text { Tableau } \\
\text { (Moyenn } \\
\text { ponica à } \\
\text { (mean } \pm \text { SC }\end{array}$ & $\begin{array}{l}\text { III. Par } \\
\text { 土écart-typ } \\
\text { semaine } \\
\text { measured in }\end{array}$ & $\begin{array}{l}\text { ètres m } \\
\text { mesurés } \\
\text { l'âge (Morp } \\
\text { turnix japoni }\end{array}$ & $\begin{array}{l}\text { pho-p } \\
\text { hez Co } \\
\text {-weight } \\
\text { at } 8 \text { wee }\end{array}$ & $\begin{array}{l}\text { ndéraux } \\
\text { urnix ja- } \\
\text { arameters } \\
\text { s of age). }\end{array}$ \\
\hline Trait & & $x e$ & $\begin{array}{c}\text { Valeur } \\
\text { de t }\end{array}$ & Sig. \\
\hline & $\begin{array}{l}\text { Mâles } \\
(n=42)\end{array}$ & $\begin{array}{c}\text { Femelles } \\
(n=58)\end{array}$ & & \\
\hline $\begin{array}{l}\text { Poids vif } \\
\text { (g) }\end{array}$ & $162,5 \pm 14,3$ & $190,62 \pm 16,5$ & 8,86 & $P<0,001$ \\
\hline $\begin{array}{l}\text { Longueur } \\
\text { corps }(\mathrm{cm})\end{array}$ & $13,00 \pm 0,75$ & $13,39 \pm 0,83$ & 2,33 & 0,02 \\
\hline $\begin{array}{l}\text { Envergure } \\
(\mathrm{cm})\end{array}$ & $31,58 \pm 1,21$ & $33,15 \pm 1,5$ & 5,56 & $P<0,001$ \\
\hline $\begin{array}{l}\text { Longueur } \\
\text { patte }(\mathrm{cm})\end{array}$ & $3,48 \pm 0,27$ & $3,52 \pm 0,25$ & 0,61 & 0,57 \\
\hline $\begin{array}{l}\text { Largeur } \\
\text { thoracique } \\
\text { (cm) }\end{array}$ & $5,07 \pm 0,44$ & $5,30 \pm 0,24$ & 2,72 & 0,008 \\
\hline $\begin{array}{l}\text { Longueur } \\
\text { bec }(\mathrm{cm})\end{array}$ & $1,28 \pm 0,08$ & $1,28 \pm 0,08$ & 0,11 & 0,9 \\
\hline
\end{tabular}

supérieures chez les animaux nourris avec un régime dosant $24 \%$ de $\mathrm{PB}$ par rapport à un régime moins riche $(22,08 \%$ de $\mathrm{PB})$. Par contre, aucun effet du niveau protéique de la ration sur les performances de croissance n'a été mis en évidence dans les travaux de Kouatcho et al. (2015) et Djouvinov et Mihailov (2005).

Les écart-types enregistrés pour le poids vif hebdomadaire indiquent une forte hétérogénéité de celui-ci, plus marquée notamment à la 2ème semaine (22,3\%). Ces résultats recoupent ceux rapportés par Berrama et al. (2011). Entre la 2ème et la 3ème semaine d'âge, le GMQ est passé de 2,07 à 4,56g, indiquant une croissance extrêmement rapide au cours de cette période. Le même constat a été fait par Özbey et al. (2006). Dauda et al. (2014) ont cependant observé une vitesse de croissance plus élevée entre la 4ème et la 5ème semaine d'âge.

Au cours de la période de finition, l'oiseau a multiplié sa prise alimentaire quotidienne par 1,5 par rapport à la période de démarrage. Parallèlement, il y'a eu un accroissement de la vitesse de croissance. Les cailleteaux ont, en effet, atteint des GMQ respectivement de 4,56 et 4,62 g/j durant la 3ème et la 4ème semaine de leur vie. Par contre, un ralentissement de la croissance a été constaté au cours de la 5ème semaine, alors qu'il n'y a pas eu de régression au niveau de l'ingéré alimentaire, l'indice de consommation a, par conséquent, été fortement détérioré $(+0,88)$. Ces résultats confirment les observations faites auparavant (Vatsalya and Arora, 2014; Nasrollah, 2009). Durant la 6ème semaine, l'indice de consommation n'a pas été suffisamment amélioré $(-0,68)$ pour compenser le retard de croissance accumulé au cours de la semaine précédente.

\section{PARAMĖTRES MORPHO-PONDÉRAUX DES ANIMAUX}

Dans cette étude, le dimorphisme sexuel chez Coturnix japonica a été constaté à partir de la 3ème semaine d'âge; la femelle présentait une poitrine claire tachetée tandis que le mâle était reconnu par un plastron roux sans taches, mais aussi par son comportement agressif, nettement remarquable. Les données sur le poids vif

Tableau IV. Corrélations de Pearson entre les paramètres morpho-pondéraux mesurés chez Coturnix japonica. (Les mâles: au-dessus de la diagonale; $\mathrm{n}=42$; Les femelles: au-dessous de la diagonale; $\mathrm{n}=58$ ) (Pearson correlations between the measured morpho-weight parameters in Coturnix japonica. (males: above the diagonal; $n=42$; Females: below the diagonal; $n=58$ ).

\begin{tabular}{lcccccc}
\hline & PV & LC & LT & ENV & LP & LB \\
\hline PV & 1 & $0,51^{\text {** }}$ & $0,46^{\text {** }}$ & $0,20^{\text {ns }}$ & $0,25^{\text {ns }}$ & $-0,10^{\text {ns }}$ \\
LC & $0,39^{\text {** }}$ & 1 & $0,28^{\text {ns }}$ & $0,44^{\text {** }}$ & $-0,05^{\text {ns }}$ & $0,08^{\text {ns }}$ \\
LT & $0,23^{\text {ns }}$ & $0,15^{\text {ns }}$ & 1 & $0,38^{*}$ & $-0,14^{\text {ns }}$ & $-0,21^{\text {ns }}$ \\
ENV & $0,20^{\text {ns }}$ & $0,01^{\text {ns }}$ & $0,22^{\text {ns }}$ & 1 & $-0,13^{\text {ns }}$ & $-0,04^{\text {ns }}$ \\
LP & $0,13^{\text {ns }}$ & $-0,05^{\text {ns }}$ & $0,16^{\text {ns }}$ & $0,08^{\text {ns }}$ & 1 & $0,33^{*}$ \\
LB & $-0,03^{\text {ns }}$ & $0,05^{\text {ns }}$ & $0,03^{\text {ns }}$ & $-0,05^{\text {ns }}$ & $-0,21^{\text {ns }}$ & 1 \\
\hline
\end{tabular}

PV : poids vif ; LC : longueur du corps ; LT : largeur thorax; ENV : envergure ; LP : longueur de la patte ; LB : longueur du bec. Les valeurs sont significativement différentes de zéro : " $P<0.05$; "* $P<0.01$; ns : les valeurs ne sont pas significativement différentes de zéro $(P \geq 0,05)$. 
Tableau V. Performances de ponte et d'éclosion de la caille Coturnix coturnix japonica (moyenne \pm écart-type) (Spawning and hatching performance of Coturnix coturnix japonica Quails (mean \pm SD).

\begin{tabular}{|c|c|c|c|c|c|}
\hline Semaines & Sem9 & Sem10 & Sem11 & Sem12 & Total \\
\hline Effectif présent femelles & $n=45$ & $n=45$ & $n=44$ & $n=44$ & $n=44$ \\
\hline Nombre œufs pondu/femelle & $3,46 \pm 0,09$ & $3,57 \pm 0,07$ & $3,79 \pm 0,11$ & $3,75 \pm 0,06$ & $14,58 \pm 0,08$ \\
\hline$\%$ de ponte & 49,5 & 51,1 & 54,2 & 53,6 & 52,1 \\
\hline \% d'œuf fêlés et/ou souillés & 3,8 & 3,72 & 2,39 & 2,42 & 3,08 \\
\hline Poids de l'œuf à incuber & $10,9 \pm 1,1$ & $11,8 \pm 0,5$ & $11,1 \pm 0,34$ & $11,6 \pm 0,45$ & $11,3 \pm 0,41$ \\
\hline \% éclosion & 89 & 81 & 90 & 78 & - \\
\hline$\%$ viabilité & 86 & 68 & 73 & 79 & - \\
\hline Poids cailleteau à la naissance & $9,2 \pm 0,85$ & $10,2 \pm 0,5$ & $9,9 \pm 0,8$ & $10,1 \pm 1,1$ & $9,85 \pm 0,65$ \\
\hline
\end{tabular}

et les paramètres de mensuration des oiseaux à 8 semaines d'âge sont présentées dans le tableau III. Les femelles pèsent significativement plus que les mâles $(+28 \mathrm{~g}, \mathrm{P}<0,001)$. Cette supériorité ne se manifeste généralement qu'à partir de la 2ème semaine (Devi et al., 2010; Berrama et al., 2011; Rathert et al., 2017), quelquefois à la 4ème semaine d'âge (Ojo et al., 2014; Victoria et al., 2014; Alasahan et Copur, 2016). Le dimorphisme sexuel a également été observé pour les paramètres de taille et de conformation, liés notamment à la longueur $(+0,39 \mathrm{~cm}, \mathrm{P}<0,05)$, l'envergure $(+1,55 \mathrm{~cm}, \mathrm{P}<0,001)$ et la largeur thoracique $(+0,23 \mathrm{~cm}, \mathrm{P}<0,01)$. Les valeurs obtenues dans notre étude sont approximativement similaires à celles trouvées par Gambo et al. (2014) mais inférieures à celles rapportées par Olawumi (2015).

Les corrélations phénotypiques entre les mensurations corporelles chez les mâles et les femelles sont présentées dans le tableau IV. Les valeurs sont comprises entre 0,12 et 0,96. Chez les mâles, le poids vif a été positivement corrélé avec la longueur du corps $(+0,51$, $\mathrm{P}<0,01)$, et la largeur du thorax $(+0,46, \mathrm{P}<0,01)$. Une corrélation modérée a été observées entre l'envergure et la longueur du corps $(+0,44, \mathrm{P}<0,01)$. Chez les femelles, hormis la relation entre le poids vif et la longueur

Tableau VI. Poids et paramètres de conformation externe de l'œuf de Coturnix japonica en fonction de la densité des oiseaux dans les cages $(A=20$ oiseaux/cage ; $\mathrm{B}=20$ oiseaux/cage), (Moyenne \pm écarttype) (Weights and parameters for the external conformation of the egg of Coturnix japonica according to the density of the birds in the cages $(A=20$ birds/cage; $B=20$ birds/cage), (mean $\pm S D$ ).

\begin{tabular}{|c|c|c|c|c|c|}
\hline \multirow[t]{2}{*}{ Paramètres } & \multicolumn{2}{|c|}{ Densité (sujet/cage) } & \multirow[b]{2}{*}{$\begin{array}{l}\text { T et } \\
\text { Sig. }\end{array}$} & \multirow[b]{2}{*}{ Total } & \multirow[b]{2}{*}{$\begin{array}{l}\text { CV } \\
(\%)\end{array}$} \\
\hline & $A(n=80)$ & $B(n=70)$ & & & \\
\hline $\begin{array}{l}\text { Poids } \\
\text { entier (g) }\end{array}$ & $11,7 \pm 0,95$ & $11,3 \pm 0,8$ & $3,18^{* *}$ & $11,54 \pm 0,93$ & 8,14 \\
\hline $\begin{array}{l}\text { Longueur } \\
(\mathrm{cm})\end{array}$ & $3,26 \pm 1,37$ & $3,23 \pm 1,41$ & $1,57^{\mathrm{ns}}$ & $3,25 \pm 1.39$ & 4,31 \\
\hline $\begin{array}{l}\text { Largeur } \\
(\mathrm{cm})\end{array}$ & $2,55 \pm 0,7$ & $2,51 \pm 0,6$ & 3,96 & $2,54 \pm 0,66$ & 2,63 \\
\hline $\begin{array}{l}\text { Indice de } \\
\text { forme }\end{array}$ & $78,4 \pm 3,04$ & $78,0 \pm 3,3$ & $0,75^{\text {ns }}$ & $78,21 \pm 3,15$ & 4,03 \\
\hline
\end{tabular}

Les différences entre les moyennes sont significatives au seuil: ${ }^{* *} \mathrm{P}<0,01 ;{ }^{* * *} \mathrm{P}<0,001$; ns: différences non significatives entre les moyennes $(P \geq 0,05)$. du corps $(+0,39, \mathrm{P}<0,01)$, les autres paramètres mesurés semblent être indépendants les uns des autres. D'autres mesures devront, néanmoins, être réalisées pour voir comment il serait possible de modifier la conformation par la sélection.

En considérant la totalité du cycle d'engraissement de 8 semaines, Oja et al. (2014) ont rapporté des valeurs comprises entre $-0,01$ et 0,70 ; selon ces mêmes auteurs, la plus forte relation enregistrée $(+0,61, \mathrm{P}<0,01)$ en fin d'élevage a été obtenue entre le poids vif et le tour de poitrine. Dans une étude précédente, (Ferrouk et al., 2015) ont rapporté de fortes corrélations allant de 0,90 à 0,99 entre l'âge du cailleteau en croissance et certains paramètres de poids, de conformation et de reproduction.

Plus généralement, les valeurs faibles à modérées obtenues dans notre étude peuvent être attribuées au nombre réduit d'animaux. Des données portant sur un effectif plus important d'animaux, notamment pendant la croissance, seront nécessaires pour éventuellement définir des modèles de prédiction du poids vif à partir des mensurations corporelles.

\section{Performances de PONTE}

Les performances de ponte enregistrée durant 4 semaines sont présentées dans le tableau V. Après la mise en cage, la mortalité a été de $2,2 \%$ durant 4 semaines. Pendant cette période, la caille a, en moyenne, pondu 14,6 œufs. L'intensité de ponte a été donc de $52,1 \%$, en moyenne; celle-ci elle est passée de 49,5\% au cours de la 9ème semaine à 53,6 \% durant la 12ème semaine d'âge, ce qui correspond à une augmentation de $4,1 \%$. Karousa et al. (2015) ont rapporté un taux de ponte compris entre $46,6 \%$ et $63,5 \%$ chez la caille âgée de 6 semaines menée en captivité à raison de 12 oiseaux/cage. Dikmen et Ipek (2006) ont, par ailleurs, mis en évidence une forte influence de la longueur des pattes sur les performances de ponte, traduite par une précocité à l'entrée en ponte (- 2,4 jours, en moyenne) et une meilleure production d'œufs (+14,7\%, en moyenne) chez les femelles ayant des tarses plus allongés par rapport à celles aux pattes plus courtes.

Le poids moyen de l'œuf à incuber obtenu dans notre essai a été de $11,3 \mathrm{~g}$, il est donc tout à fait conformes aux valeurs recommandées (ITELV). Ce poids est cependant inférieur à ceux obtenues par Sangili- 
Tableau VII. Qualité interne de l'oeuf de Coturnix japonica en fonction de la densité des oiseaux dans les cages $(A=20$ oiseaux/cage; $B=20$ oiseaux/cage), (Moyenne \pm écart-type) (Internal quality of the egg of Coturnix japonica according to the density of the birds in the cages $(A=$ 20 birds/cage; $B=20$ birds/cage), (mean $\pm S D$ ).

\begin{tabular}{|c|c|c|c|c|c|}
\hline & Densité (o & seaux/cage) & & & \\
\hline Paramètre & $A(n=80)$ & $B(n=70)$ & $\begin{array}{l}\text { T et } \\
\text { Sig. }\end{array}$ & $\begin{array}{c}\text { Total } \\
(n=150)\end{array}$ & $\begin{array}{l}\text { CV } \\
(\%)\end{array}$ \\
\hline $\begin{array}{l}\text { Poids } \\
\text { Coquille } \\
\text { (g) }\end{array}$ & $1,4 \pm 0,23$ & $1,5 \pm 0,25$ & $1,25^{\mathrm{ns}}$ & $1,47 \pm 0,24$ & 16,4 \\
\hline $\begin{array}{l}\text { Poids } \\
\text { Albumen } \\
\text { (g) }\end{array}$ & $5,8 \pm 0,65$ & $5,7 \pm 0,60$ & $0,72^{\text {ns }}$ & $5,81 \pm 0,63$ & 10,8 \\
\hline $\begin{array}{l}\text { Poids } \\
\text { Vitellus (g) }\end{array}$ & $3,49 \pm 0,41$ & $3,51 \pm 0,42$ & $0,30^{\text {ns }}$ & $3,50 \pm 0,41$ & 11,8 \\
\hline $\begin{array}{l}\text { Proportion } \\
\text { Coquille } \\
(\%)\end{array}$ & $12,3 \pm 1,9$ & $13,3 \pm 2,3$ & $2,81^{* *}$ & $12,82 \pm 2.14$ & 16,7 \\
\hline $\begin{array}{l}\text { Proportion } \\
\text { Albumen } \\
(\%)\end{array}$ & $49,6 \pm 3,02$ & $51,1 \pm 3,67$ & $2,70^{* *}$ & $50,32 \pm 3.41$ & 6,7 \\
\hline $\begin{array}{l}\text { Proportion } \\
\text { Vitellus } \\
(\%)\end{array}$ & $29,7 \pm 2,47$ & $31,1 \pm 2,94$ & $3,27^{* *}$ & $30,40 \pm 2.78$ & 9,2 \\
\hline $\begin{array}{l}\text { Hauteur } \\
\text { Albumen } \\
(\mathrm{cm})\end{array}$ & $0,39 \pm 0,05$ & $0,38 \pm 0,05$ & $0,62^{\text {ns }}$ & $0,38 \pm 0,05$ & 13,6 \\
\hline $\begin{array}{l}\text { Hauteur } \\
\text { Vitellus } \\
(\mathrm{cm})\end{array}$ & $1,12 \pm 0,08$ & $10,10 \pm 0,07$ & $1,21^{\mathrm{ns}}$ & $1,11 \pm 0,08$ & 7,04 \\
\hline $\begin{array}{l}\text { Diamètre } \\
\text { Vitellus } \\
(\mathrm{cm})\end{array}$ & $2,26 \pm 0,12$ & $2,27 \pm 0,11$ & $0,46^{\mathrm{ns}}$ & $2,27 \pm 0,11$ & 5,03 \\
\hline $\begin{array}{l}\text { Indice } \\
\text { Vitellus }\end{array}$ & $49,6 \pm 4,44$ & $48,7 \pm 4,5$ & $1,20^{\text {ns }}$ & $49,24 \pm 4.46$ & 9,06 \\
\hline $\begin{array}{l}\text { Unités } \\
\text { Haugh }\end{array}$ & $57,1 \pm 1,63$ & $57,8 \pm 1,54$ & $2,39^{*}$ & $57,4 \pm 1.57$ & 2,74 \\
\hline
\end{tabular}

Les différences entre les moyennes sont significatives au seuil " $P<0,05$; " $P<0,01$; ns: différences non significatives entre les moyennes $(P \geq 0,05)$ madan et al. (2012), Moula et al. (2014), et Berrama et al. (2011), respectivement de 12,$4 ; 12,3$ et $11,8 \mathrm{~g}$. Nous avons enregistré peu d'hétérogénéité pour ce paramètre; la variation est passé de $10 \%$ pendant à la 9ème semaine à moins de $4 \%$, en moyenne pendant les trois semaines suivantes. D'autre part, le taux d'éclosion moyen et la viabilité obtenus ont été respectivement de 84,5 et $76,5 \%$. La variation de ces deux paramètres durant 4 semaines a été respectivement de 5,9 et $10,1 \%$, en moyenne. Globalement, les performances enregistrées sont bonnes surtout si on tient compte du sexe ratio appliqué dans cet essai, mais aussi de la durée de stockage des œufs, relativement longue, pouvant avoir un effet dépressif sur les constituants de l'œuf et, par conséquent, sur les autres paramètres d'intérêt économique, viabilité, pouvoir d'éclosion et croissance pondérale, en particulier. Selon Roriz et al. (2016), une durée de conservation de 5 jours au maximum est fortement recommandée pour maintenir une bonne qualité de l'œuf à incuber.

En termes de poids du cailleteau à la naissance, celui-ci a été de $9,85 \mathrm{~g}$, en moyenne $(86,8 \%$ du poids initial de l'œuf). Des poids semblables compris entre 9,1 et 9,8g ont été rapportés par d'autres auteurs (Romao et al., 2009, Silva et al., 2013). Le poids obtenu dans cette étude est cependant supérieur à 8,2g enregistré par Metin Sezer (2007) chez la caille pondeuse, et celui de 9,41g obtenu chez deux variétés (Noire et Marron) sélectionnées pour leur poids élevé à l'âge de 4 semaines (Devi et al., 2010).

Plus génarlement, des écarts plus ou moins importants ont été constatés entre les résultats des auteurs pour ce qui concerne la qualité globale des pontes. Celle-ci est influencée par un mélange de facteurs génétiques et non génétiques (âge, sexe ratio, alimentation, etc). Islam et al. (2014) situent le taux d'éclosion entre $31 \%$ et $71 \%$. Selon ces mêmes auteurs, Coturnix japonica a montré une nette supériorité $(+26 \%)$ par rapport à trois autres phénotypes de caille Blanc, Marron et Noir. En appliquant un sexe ratio de 1:2 et 1:3, Karousa et al. (2015) ont rapporté des taux d'éclosion respectivement de $49 \%$ et $52 \%$. Moraes et al. (2008) ont obtenu dans leurs travaux un taux d'éclosion de $77 \%$ pour des œufs incubés en position horizontale (avec retournement)

Tableau VIII. Corrélations de Pearson entre certains paramètres internes et externes de l'œuf de Coturnix coturnix japonica $(\mathrm{n}=150)$ (Pearson correlations between some internal and external parameters of the egg of Coturnix Coturnix japonica $(n=150)$.

\begin{tabular}{|c|c|c|c|c|c|c|c|c|c|}
\hline & 1 & 2 & 3 & 4 & 5 & 6 & 7 & 8 & 9 \\
\hline 1-Poids de l'œuf entier & 1 & $0,23^{* *}$ & $0,78^{* * *}$ & $0,63^{* * *}$ & 0,01 & $0,28^{* * *}$ & $0,46^{* * *}$ & $0,75^{* * *}$ & $0,86^{* * *}$ \\
\hline 2-Poids de coquille & & 1 & $0,22^{* *}$ & $0,34^{* * *}$ & $-0,04^{\mathrm{ns}}$ & $0,17^{\star}$ & $0,06^{\text {ns }}$ & $0,24^{* * *}$ & $0,1^{\text {ns }}$ \\
\hline 3-Poids du blanc & & & 1 & $0,43^{\star * \star}$ & $0,04^{\mathrm{ns}}$ & $0,19^{* *}$ & $0,22^{* *}$ & $0,60^{* * *}$ & $0,67^{* * *}$ \\
\hline 4-Poids du jaune & & & & 1 & $-0,07^{\mathrm{ns}}$ & $0,29^{* * *}$ & $0,57^{\star * \star}$ & $0,59^{* * *}$ & $0,50^{* * *}$ \\
\hline 5-Hauteur du blanc & & & & & 1 & $0,06^{\text {ns }}$ & $-0,21^{* *}$ & $-0,03^{\text {NS }}$ & $0,03^{\text {ns }}$ \\
\hline 6-Hauteur du jaune & & & & & & 1 & $-0,10$ & $0,23^{* *}$ & $0,23^{* *}$ \\
\hline 7-Diamètre du jaune & & & & & & & 1 & $0,47^{* * *}$ & $0,34^{* * *}$ \\
\hline 8-Longueur de l'œuf & & & & & & & & 1 & $0,39^{* * *}$ \\
\hline 9-Largeur de l'œuf & & & & & & & & & 1 \\
\hline
\end{tabular}

Les valeurs sont significativement différentes de zéro: ${ }^{* *} \mathrm{P}<0,01 ;{ }^{* *} \mathrm{P}<0,001$; ${ }^{\mathrm{ns}}$ : les valeurs ne sont pas significativement différentes de zéro $(P \geq 0,05)$. 
contre un taux d'éclosion de $65 \%$ pour ceux incubés verticalement, petit bout vers le bas.

\section{Caractéristiques de l'oeuf de Coturnix coturnix JaponicA}

Les tableaux VI et VII contiennent une comparaison des paramètres morpho-pondéraux mesurés chez les œufs issus des femelles maintenues à deux densités différentes (groupe A : 20 oiseaux/cage ; groupe B : 25 oiseaux/cage). Le poids moyen de l'œuf a été de 11,5g avec une variation de $8,14 \%$. Ce poids est comparable à ceux obtenus par d'autres auteurs (Smamri et al., 2014; Hrnčár et al., 2014). Les valeurs obtenues dans cet essai sont cependant inférieurs à celles rapportées par Genchev (2013) chez deux souches différentes de Coturnix japonica, Pharaoh et Manchurian Golden respectivement de 12,4 et $13,0 \mathrm{~g}$. Les valeurs moyennes pour la longueur et la largeur des œufs ont été respectivement de 3,25 et 2,54 cm, soit un indice de forme moyen de 78,2 , indiquant des œufs de gros calibre. Hrnčár et al. (2014) ont obtenu un indice de forme similaire mais sur des œufs plus lourds $(+1,56 \mathrm{~g})$; ces derniers contiennent, en effet, relativement plus d'albumen et de vitellus pour la même quantité de coquille. La largeur de l'œuf a présenté la variabilité la plus faible $(2,6 \%)$ parmi tous les paramètres mesurés. Des résultats similaires ont été rapportés par Kul et Seker (2004). Comparés aux pondeuses du groupe $\mathrm{B}$, celles du groupe $\mathrm{A}$ produisent des œufs plus lourds $(+0,4 \mathrm{~g})$ et plus larges $(+0,04 \mathrm{~cm})$ sans toutefois présenter de différence en termes de calibre. Le poids du blanc et celui du vitellus ont été respectivement de 5,8 et 3,5g, correspondant à des proportions (par rapport au poids de l'œuf entier) respectivement de 50,3 et 30,4\%. Ces 2 composants ont tendance à diminuer avec l'âge de la pondeuse (Nazligul et al., 2001). Etant liée à un plus fort taux de matière

Tableau IX. Valeurs propres et pourcentages de la variance expliquée pour neuf paramètres mesurés sur les œufs de caille Coturnix coturnix japonica (Eigenvalues and percentages of variance explained for nine parameters measured on quail eggs Coturnix coturnix japonica).

\begin{tabular}{lccc}
\hline Traits $^{1}$ & \multicolumn{3}{c}{ Composante $^{1}$} \\
\hline & $\mathrm{CP}_{1}$ & $\mathrm{CP}_{2}$ & $\mathrm{CP}_{3}$ \\
\cline { 2 - 4 } Poids de l'œuf entier & $\mathbf{0 , 9 5 7}$ & 0,043 & 0,156 \\
Poids de coquille & 0,083 & 0,209 & $\mathbf{0 , 7 9 1}$ \\
Poids du blanc & $\mathbf{0 , 8 2 3}$ & $-0,121$ & 0,136 \\
Poids du jaune & 0,655 & 0,374 & 0,371 \\
Hauteur du blanc & 0,118 & $\mathbf{- 0 , 7 4 3}$ & $-0,059$ \\
Hauteur du jaune & 0,209 & $-0,307$ & 0,678 \\
Diamètre du jaune & 0,528 & 0,666 & $-0,151$ \\
Longueur de l'œuf & $\mathbf{0 , 7 3 4}$ & 0,228 & 0,239 \\
Largeur de l'œuf & $\mathbf{0 , 8 6 0}$ & $-0,092$ & 0,006 \\
Valeur propre & 3,990 & 1,283 & 1,070 \\
\% de la variance expliquée & 44,35 & 14,26 & 11,88 \\
par l'axe & & & \\
\% cumulé de la variance & 44,35 & 58,61 & 70,50 \\
expliquée par l'axe & & &
\end{tabular}

${ }^{1}$ Arbitrairement les valeurs spérieures à 0,7 indiquant des contributions importantes sur les axes sont présentées en caractère gras. sèche dans l'oeuf et aussi à un apport plus important d'acides gras essentiels, une proportion de jaune plus élevée peut être considérée comme favorable du point de vue de la valeur nutritive de l'œuf (Benabdeljelil et Mérat, 1995). Quant au poids de la coquille, celui-ci a été de $1,4 \mathrm{~g}$, soit une proportion de $12,8 \%$. Le rapport du poids du jaune au poids du blanc a été de 60,7 , en moyenne. L'épaisseur du blanc a été de $0,38 \mathrm{~cm}$. Quant à l'indice vitellus, qui permet de juger de la consistance du jaune, il a été de 49,2. Ce paramètre a tendance à diminuer avec l'âge de la pondeuse et la durée de stockage (Moula et al., 2014; González Sánchez, 2009 ; Tilki et Saatci, 2004). Il apparaît que les œufs issus des deux groupes contiennent approximativement la même quantité de coquille, de jaune et de blanc, mais en termes de proportions, les femelles du groupe B ont montré leur supériorité par rapport à leurs homologues du groupe A. Il en de même de la fraîcheur de l'œuf, déterminée ici par les unités Haugh. Pour les autres paramètres, les valeurs moyennes des deux groupes ont été équivalentes. Ces résultats nécessitent tout de même d'être confirmés sur un effectif plus important en adoptant un protocole plus rigoureux.

\section{CORRÉLATIONS PHÉNOTYPIQUES ENTRE LES PARAMÈTRES DE L'OEUF}

Les corrélations phénotypiques entre les caractères internes et externes mesurés sur 150 œufs de caille sont présentées dans le tableau VIII. Les valeurs obtenues sont comprises entre 0,01 et 0,86 . Le poids de l'œuf entier est fortement corrélé avec les paramètres de conformation de l'œuf (longueur et largeur) (respectivement, $r=0,86$ et $r=0,75, P<0,001)$, le poids du blanc $(r=0,78$, $\mathrm{P}<0,001)$ et celui du vitellus $(\mathrm{r}=0,63, \mathrm{P}<0,001)$. Par contre, une relation de faible intensité $(\mathrm{r}=0,23, \mathrm{P}<0,01)$ a été observée avec le poids de la coquille. Comparé au poids du vitellus, celui du blanc a été plus fortement lié à la longueur et la largeur de l'œuf (respectivement, $r=0,60$ et $r=0,67, P<0,001)$.

Les corrélations obtenues présentent certainement un réel intérêt puisqu'elles doivent nous permettre de prédire la qualité interne de l'œuf à partir de certains paramètres externes, facilement mesurables sans devoir casser les œufs (non-invasifs) d'une part, et d'améliorer la qualité de l'œuf par la sélection génétique, d'autre part. Des constatations tout à fait comparables ont été rapportées par d'autres auteurs (Alkan et al., 2015 ; Oczelik, 2002; Kul et Seker, 2004). Selon Alkan et al. (2008), la largeur de l'œuf constitue le critère le plus précis pour prédire la hauteur du vitellus. En outre, chez la pintade, Onunkwo et Okoro (2015) ont proposé des équations linéaires de prédiction du poids du blanc et celui du jaune à partir du poids de l'œuf entier ; les modèles établis par ces auteurs, ont présenté des coefficients de détermination compris entre 43,2 et $68,6 \%$.

Les résultats de l'analyse en composantes principales des corrélations phénotypiques entre les paramètres de l'œuf mesurés sont présentés dans le tableau IX. Le déterminant de la matrice des corrélations entre les paramètres de l'œuf a été de 0,003 tandis que l'indice $\mathrm{KMO}$ a été de $0,66\left(\chi^{2}=826,7 ; \mathrm{P}<0,001\right)$. Dans le cas présent il semble satisfaisant d'interpréter les résultats des trois composantes (à valeurs propres supérieures 
à 1) de plus grandes variances et de négliger les autres, d'autant plus que les variances de ces dernières sont relativement faibles par rapport à la variance totale (Dahloum, 2017). Ainsi, les trois composantes principales retenues CP1, CP2 et CP3 expliquent respectivement $44,35 \%, 14,26 \%$ et $11,90 \%$ de la variance totale, soit une variance cumulée de 70,5\%.

La première composante qui rend compte du maximum de la variance totale est fortement représentée par le poids de l'œuf entier, le poids de l'albumen, la longueur et la largeur de l'œuf. La deuxième composante indique une opposition entre la hauteur du blanc et le diamètre du jaune, même si la contribution de ce dernier sur l'axe paraît peu intense. Quant à la troisième composante, celle-ci est presque exclusivement représentée par le poids de la coquille et peut donc être considérée comme "facteur spécifique" pour ce paramètre. Plus généralement, les trois premières composantes extraites semblent être suffisantes pour expliquer les variations en termes de conformation et de composition externe de l'œuf. L'interprétation des composantes peut bien sûr être différente selon la nature et le nombre de variables étudiées.

\section{CONCLUSION}

Les performances de croissance enregistrées chez le cailleteau Coturnix japanica sont dans l'ensemble intéressantes quoiqu'en deça des résultats enregistrés chez les souches sélectionnées ou celles menées en élevage standard. Pour le poids vif, sélectionner à 2 semaines permet d'escompter un gain substantiel, en raison de la forte variabilité de ce caractère à cet âge. Les données concernant la ponte, disponibles ici, ne nous permettent pas présentement d'évaluer de manière précise le niveau des performances de ponte de la caille. En ce qui concerne la qualité des œufs, celle-ci pourrait, dans tous les cas, être améliorée par la sélection génétique, en raison des corrélations modérées à fortes estimées entre les variables. Mais avant cela, des études plus spécifiques consistant à vérifier l'influence d'autes facteurs, âge, température, alimentation, sur les performance de la caille seront nécessaires.

\section{REMERCIEMENTS}

Les auteurs de cet article remercient vivement le Centre Cynégétique de Zéralda (Algérie) pour l'aide matérielle et les facilités qui nous ont été accordées.

\section{BIBLIOGRAPHIE}

Alasahan, S \& Copur, AG 2016, 'Hatching Characteristics and Growth Performance of Eggs with Different Egg Shapes', Brazilian Journal of Poultry Science. vol 18, no 1, pp. 001-008, http://dx.doi. org/10.1590/1516-635×1801001-008.

Alkan, S, Karabag, K, Galic, A, \& Balcioglu, S 2008, 'Predicting yolk height, albumen lenght, eggshell weight, egg shape index, eggshell thickness, egg surface area of Japanese quails using various eggs traits as regressors'. International Journal of Poultry Science, vol 7, no 1, pp- 85-88.

Alkan, S, Galiç, A, Karsli T, \& Karabağ, K 2015, 'Effects of egg weight on egg quality traits in partridge (Alectoris Chukar). Journal of Applied
Animal Research, vol. 43, no. 4, pp,450-456, http://dx.doi.org/10 .1080/09712119.2014.980419

Angrand, A 1986, 'Contribution à l'étude de la qualité commerciale des œufs de consommation de la région de Dakar (Sénégal)', Thèse de doctorat, E, I, S, M, Y, 1986, $158 \mathrm{pp}$,

Bagh, J, Panigrahi, B, Panda, N, Pradhan, CR, Mallik BK, Majhi B, \& Rout, SS 2016 . 'Body weight, egg production, and egg quality traits of gray, brown, and white varieties of Japanese quail (Coturnix coturnix japonica) in coastal climatic condition of Odisha', Veterinary World. vol, 9, no. 8, pp. 832-836.

Benabdeljelil, K et Mérat, P 1995, 'Comparaison de types génétiques de poules pour une production d'oeufs locale: F1 (Fayoumi x Leghorn) et croisement terminal ISA au Maroc'. Annales de zootechnie, no 44, pp. 313-318.

Berrama, Z, Mefti, H, Kaidi, R, et Souames, S 2011, 'Caractérisation zootechnique et paramètres génétiques des performances de croissance de la caille Japonaise Coturnix japonica élevée en Algérie', Livestock Research for Rural Development, vol, 23, no 1.

Berto, BP, Borba, HR, Lima VM, Flausino, W, Teixeira-Filho, WL, \& Lopes, CWG 2013, 'Eimeria spp. from Japanese quails (Coturnix japonica): new characteristic features and diagnostic tools'. Pesquisa Veterinária Brasileira, vol, 33, no 12, pp.1441-1447.

Dahloum, L 2017, 'Caractérisation phénotypique de la poule locale (gallus gallus) dans le Nord-Ouest algérien. Gènes majeurs et thermotolerance', Thèse de Doctorat en sciences, Université de Mostaganem, Algérie, $\mathrm{p} 140$.

Dauda, G, Momoh, OM, Dim, NI, \& Ogah, DM 2014, 'Growth, production and reproductive performance of Japanese quails (Coturnix coturnix japonica) in humid environment', Egyptian Poultry Science, vol. 34, no ll, pp. 381-395.

Devi, KS, Gupta, BR, Prakash, MG, Qudratullah, S, \& Reddy, AR 2010, 'Genetic studies on growth and production traits in two strains of Japanese quails'. Tamilnadu Journal of Veterinary and Animal Sciences, vol. 6, no. 5, pp. 223-230.

Dikmen BY, \& Ipek, A 2006, 'The Effects of Shank Length on egg production and egg quality traits of Japanese Quails (Coturnix coturnix japonica)'. 12th European Poultry Conference, Verona, Italy, 10-14.

Djouvinov, D and Mihailov, R, 2005, 'Effect of low protein level on performance of growing and laying Japanese quails (Coturnix coturnix Japonical', Bulgarian Journal of Veterinary Medicine, vol 8, no 2, pp. 91- 98.

FAO, 2009, 'Comment nourrir le monde en 2050', Forum d'experts de haut niveau, Rome, 12 et 13 octobre, p. 2.

Ferrouk, M, Boukenaoui, N, Smaili, I, Abd Al samad, I et Exbrayat, J M 2015, 'Etude morphométrique et histologique du testicule de la caille du japon (Coturnix japonica) au cours de la croissance post-natale', Bulletin de la Siciété Zoologique de France, vol, 140, no 1, pp. 45-60.

Gambo D, Momoh, OM, Dim, N I, \& Kosshak AS 2014, 'Body parameters and prediction of body weight from linear body measurements in Coturnix quail'. Livestock Research for Rural Development. Vol 26, Article \#1 10. Retrieved October 18, 2017, from http://www.Irrd. org/lrrd26/6/daud26110.html.

Genchev, A 2012, 'Quality and composition of japanese quail eggs (Coturnix japonica)', Trakia Journal of Sciences, vol, 10, no. 2, pp. 91-101.

Gerken, M and Mills, AD 1993, 'Welfare of domestic quail In "Savory, CJ, Hughes BO, (Eds) Fourth European Symposium on poultry welfare", Edinburgh University federation for animal welfare Potters Bar, pp. $158-176$.

González Sánchez, JF, Chamorro Ramirez, FH, \& Hernández Unzon, H 2009, ' Physicochemical Changes in Quail Eggs (Coturnix coturnix japonica) after Storage at Different Temperatures. Journal of Applied Animal Research, vol 35, pp. 177-180.

Hantanirina, HI, Rabearimisa, RN, \& Rakotozandriny JN 2013, 'Domestication de la caille à Madagascar: Cas de la Caille Japonaise "Coturnix japonica' (Termminck \& Schlegel, 1849), 3 Akon'ny Ala $N^{\circ} 32$, ESSA-Forêt, www, Essa-forets,org. 
Hrnčár C, Hanusová, E, Hanus, A, \& Bujko, J 2014, 'Effect of genotype on egg quality characteristics of Japanese quail (Coturnix japonica)'. Slovak Journal of Animal Science, vol 47, no 1, pp. 6-11.

Islam, MS, Faruque, S, Khatun, H, \& Islam, MN 2014, 'Effects of quail genotypes on hatchability traits, body weight and egg production'. Journal of Bangladesh Academy of Sciences, vol, 38, no 2, 219-224.

Jatoi, AS, Mehmood, S, Hussain, J, Ishaq, HM, Abbas, Y, Akram, M 2015, 'Comparison of Six-Week Growth Performance in Four Different Strains of Japanese Quail (Coturnix coturnix japonica)', Sarhad Journal of Agriculture, vol. 31, no. 1, pp. 59-64.

Karousa, MM, Souad AA, Elaithy, SM, \& Eman AE 2015, 'Effect of housing system and sex ratio of quails on egg production, fertility and hatchability'. Benha Veterinary Medical Journal, vol. 28, no 2, pp. 241-247.

Kouatcho, FD, Kana, JR, Ngoula F, Nana, NFC, \& Teguia, A 2015, 'Effet du niveau de protéines brutes sur la croissance et la carcasse chez la caille (Coturnix sp) en phase de finition dans les Hautes Terres du Cameroun', Livestock Research for Rural Developmen, vol. 27, no. 8.

Kul, S, and Seker, I 2004, 'Phenotypic correlations between some external and internal egg quality traits in the Japanese quail (Coturnix coturnix japonica)' International Journal of Poultry Science, 3, pp. 400-405.

Le Bot, $\bigcirc 2014$, 'Influences maternelles prénatales chez les oiseaux nidifuges : facteurs de variation et effets sur le développement comportemental des jeunes', Thèse / Université de Rennes 1, p. 279.

Lucotte, G 1974, 'La production de la caille', Edition Vigot frères Paris $77 \mathrm{pp}$.

Moula, N, Philippe, FX, Ait Kaki, A, Touazi, L, Antoine-Moussiaux, N, et Leroy, P 2014, 'Ponte et qualité d'œufs de cailles élevées en conditions semi intensives dans l'est algérien', Archivos de Zootecnia, vol, 63, no. 244 , pp. 693-696

Narinc, D, Aaksoy, T\& Karaman, E 2010, 'Genetic parameters of growth curve parameters and weekly body weights in Japanese quails (Coturnix coturnix japonica)', Journal of Animal and Veterinary Advances, vol. 9, no. 3, pp. 501-507.

Nasrollah, V 2009. 'Growth, feed Consupmtion and Carcass Composition of Coturnix japonica, Coturnix ypsilophorus and their Reciprocal Crosses', Asian Journal of Poultry Science, vol 3, no, pp. 132-137.

Nazligul, A, Turkyilmaz, K, \& Bardakçioglu, HE 2011, 'A study on some production traits and egg quality characteristics of Japanese quail'. Turkish Journal of Veterinary and Animal Sciences, Vol, 25, pp. 1007-1013.

Oczelik M, 2002 'The phenotypic correlation among some external and internal quality characteristics in the Japanese quail eggs', Veterinary Journal ofn Ankara University, vol, 49, pp. 67-72.

Olawumi, S O 2015, 'House $x$ sex interaction effects on body weight and linear measurements of Coturnix quails', Animal and Veterinary Sciences, vol. 3, no 1, pp 18-21.doi: 10.11648/i.avs.20150301.14

Özbey O, Yildiz N, \& Esen F 2006, 'The effects of high temperature on breeding characteristics and the living strength of the Japanese quail (Coturnix Coturnix Japonica)', International journal of Poultry Science, vol. 5, no 1, pp. $56-59$.

Rathert, TÇ, Güven I, \& Üçkardeş, F 2017, 'Sex Determination of Japanese Quails (Coturnix Coturnix Japonica) using with Zoometric Measurements'. Turkish Journal of Agriculture - Food Science and Technology, vol. 5, no. 9, pp. 1002-1005.
Recoquillay J 2014, 'Architecture génétique du comportement chez la Caille Japonaise et relations avec des caractères de production', Thèse de doctorat de l'université François-Rabelais de Tours, France

Reddy PM, Reddy, VR, Reddy, CV \& Rap, PSP 1979, 'Egg weight, shape index and hatch ability in Khaki Campbell duck egg', Indian Journal of Poultry science, vol. 14, pp. 26- 31

Romao, JM, Moraes, TGV, Teixeira, RSC, Buxade, CC, Cardoso, WM 2009, 'Incubation of Japanese quail eggs at different temperatures: Hatchability, hatch weight, hatch time and embryonic mortality', Archives of Veterinary Science, vol.14, no. 3, pp.155-162.

Roriz BC, Sgavioli S, Garcia RG, Naas IA, Domingues CHF, Caldara FR, Rombola LG, Ayla CM, \& Bernnecke K 2016, 'Storage Period Affects Weight Loss of Japanese Quail Eggs', Brazilian Journal of Poultry Science, vol. 18, no. 4, pp. 589-592 http://dx.doi.org/10.1590/18069061-2015-0178.

Sangilimadan K, Rajini AR, Prabakaran, Balakrishnan, RV, \& Murugan, M 2012, 'Effect of dietary protein on layer Japanese quails (Coturnix coturnix japonica) in Tropics', Tamil Nadu Journal of Veterinary and Animal Sciences, vol. 8, no 5, pp. 271-278.

Sezer, M 2007, 'Genetic Parameters Estimated for Sexual Maturity and Weekly Live Weights of Japanese Quail (Coturnix coturnix japonica)', Asian Australasian Journal of Animal Sciences, vol, 20, no 1, pp. 19-24.

Sharifi, M R, Shargh, M S, Dastar, B, \& Hassani, S 2011, 'The effect of dietary protein levels and synbiotic on performance parameters, blood characteristics and carcass yields of Japanese quail (Coturnix coturnix Japonica), Italian Journal of Animal Science, vol. 10, no. 1, pp. 17-21, http://dx.doi.org/10.4081/ijas.2011.e4

Silva, LP, Ribeiro, JC, Crispim, AC, Silva, FG, Bonafe', CM, Silva, FF, Torres, RA 2013, 'Genetic parameters of body weight and egg traits in meat-type quail', Livestock Science, 153, pp. 27-32.

Smamri, TY, Azeroual, E, Lamtai, M, Ouldsidimoctar, Y, Benazzouz B, \& Mesfioui A 2014, Effet de sept plantes aromatiques et medicinales sur l'âge à la maturite sexuelle et sur les performances de ponte chez la caille Japonaise (Coturnix japonica)'. Illème Symposium de la recherche avicole 20-21 Octobre, Batna, Algérie.

Tilki, M, and Saatci, M 2004, 'Effects of storage time on external and internal characteristics in partridge (Alectoris graeca) eggs', Revue de Médecine Vétérinaire, vol, 155, no. 11, pp. 561-564.

Tixier-Boichard, M 1992, 'L'amélioration génétique en France: le contexte et les acteurs Les Volailles', INRA Productions animales, hors série, 35-38.

Vatsalya, V, \& Arora, KL 2011, 'Association Between Body Weight Growth and Selected Physiological Parameters in Male Japanese Quail (Coturnrix japonica)'. International Journal of Poultry Science.vol 10, no.9, pp.680-684. doi:10.3923/ijps.2011.680.684.

Victoria, O, Fayeye, TR, Ayorinde, LK, \& Olojede, H 2014, 'Relationship between Body Weight and Linear Body Measurements in Japanese Quail (Coturnix coturnix japonica)', Journal of Scientific Research, vol. 6, no 1, pp. 175-183, http://dx.doi.org/10.3329/jsr.v6i1.16368 